FACULDADE DE MEDICINA E CIRURGIA DE S. PAULO

Serviço clinico do Prof. Ovidio Pires de Campos, no Hospital Central da

Casa Santa de Misericordia de Sảo Paulo

\title{
UM CASO DE SYNDROME DE
}

\section{BROWN-SÉQUARD}

\author{
Observação dos DRS. FELICIO CINTRA PRADo e FELIPE FIGLIOLINI
}

Deparando-nos a opportunidade um caso bastante completo da syndrome de Brown-Séquard, com lesão da medulla no lado opposto ao orfficio de entrada do instrumento perfuro-cortante com o qual foi praticada, resoivemos a sua publicação, menos, muito menos pela raridade do caso, cio que pela nitidez das desordens sensitivo-motoras.

\section{OBSERVAC̣ÃO.}

A. L., lavrador, hespanhol, com 56 annos de idade, casado, morador em Santo Antonio do Pinhal.

Carecem de importancia os antecedentes hereditarios e pessoaes.

Conta o doente que na tarde do dia 15 de Abril ultimo, quando se entrietinha na arrumação das achas de uma fogueira, com o tronco curvo para a frente, foi inopinadamente aggredido por um desaffecto que the vibrou uma facada nas costas. Com a pressa da fuga, deixou o aggressor immersa na ferida a lamina da arma, emquanto o doente, sem perder a lucidez, cahia pesadamente com a perna direita paralysada.

Transportado para o leito, ahi passou a noite com intensas dôres no logar do ferimento e escarrando sangue. No dia seguinte, conduzido á Santa Casa de Piracicaba, foi submettido a medicações locaes, que consistiram em tintura de iodo e pontas de fogo, e, internamente, poçôes iestimulantes.

Teve incontinencia de urinas e só conseguiu defecar mercê de lavagens intestinaes.

Para logo os escarros hemoptoicos desappareceram e as dôres do hemithorax direito abrandaram. O membro inferior direito, inteiramente paralytico de começo, foi séde de sensações de calor e formigamento, para voltar quasi á sensibilidade normal dentro de 15 dias. 
Só no dia 10 de Maio, quando se preparava para viajar para São Paulo, notou que os movimentos no membro inferior direito eram possiveis. embora pequenos, ao passo que o membro correspondente esquerdo apresentava certa dormencia.

A. L. é de estatura regular, compleição mediana, bem conformado. mas muito emagrecido. Consciencia lucida, preferindo na eustase o rlecubito donsal.

Apparelho respiratorio - Thorax sub-globoso, com pequenos movimentos. Expansão accentuadamente menor do hemithorax esquerdo.

$\mathrm{Na}$ parede posterior do thorax (lado esquerdo), uma cicatriz profunda em fórma de $\mathrm{X}$, e para dentro, a dois diedos transversos da columna e ao nivel da 2 ' $^{\prime}$ vertebra dorsal, outra cicatriz grosseiramente linear, com 1 e meio centimetros de comprimento, dirigida de dentro para fóra e de cima para baixo. Aquella, reliquat de uma intervencão cirurgica, esta do incidente que o enfermou.

De cada lado da columna e em toda a altura do thorax, pequenas cicatrizes produzidas pela platina candente do thermocauterio.

As inspirações forçadas despertam dôr anteriormente na base do hemithorax direito.

Ligeiro augmento de frémito no lado esquerdo. A percussão revela sub-macissez em ambas as bases, principalmente á esquerda. Escuta: estertores sub-crepitantes de pequenas bolhas em todo o pulmão esquerdo e na base do direito, conjuntamente a roncos e sibillos.

Apparelho circulatorio - Ligeiro reforço da segunda bulha nos fóco's aorticos.

Apparelho digestivo e genito-urinario: - nada.

Systema nervoso: - Marcha: Passos curtos, ceifando com a perna direita. Equilibrio: perfeito.

Signal de Romberg: negativo. Noção segmentaria presente.

Paralysia incompleta do membro inferior direito.

Sensibilidade superficial -. (thermica, dolorosa e electrica):

Lado direito. a) uma fiaixa de "anesthesia que abarca o hemithorax direito, começando na parede posterior entre a 5." e a 9." vertebras dorsaes e dirigindo-se para a anterior até o concavo da axilla, compromettida até na porção mais aita. Em correspondencia da axillar anterior, o limite superior desce e passa a constituir o limite superior do restante da faixa situata mais abaixo, consoante se vê nas gravuras. Junto ao externo o limite superior dista da furcula de 11 centimetros;

b) uma faixa de hyperesthesia acima da precedente, cujo limite superior não póde ser nitidamente dermographado;

c) hyperesthesia que vae do lịmite inferior da faixa anesthesica até a extremidade do membro correspondente. Esta póde ser por 
sua vez subdividida em duas zonas de hypersensibilidade, differentes: uma, que vae da faixa anesthesica até aos 10 e meio centimetros, na qual a hyperesthesia é intensissima; e outra, dahi até a extremilade do membro, menos accentuada.

Lado esquerdo: Umina zona triangular de transição logo acima da arcada die Poupart e dahi até a extremidade do membro, hypoesthesia.

A pesquiza cuidadosa c reiterada no restante da metade esquerda, não logrou evidenciar outras desordens sensitivas.

Reflectividade:

a) superficial: ausentes os abdominaes do lado esquerdo. Presente o cremasterino de ambos os lados.

b) profunda: exaggerados em ambos os lados (achilleano $e$ rotuliano) se bem que os do direito sejam muito mais vivos.

Trepidação epileptoide da rotula e pé do lado direito: esboçada á esquerda.

Phenomeno de Babinski á direita.

Sensibilidade profunda (baresthesica, ossera, articular, etc.) presente.

Reflexos pupillares: normaes á luz e á accomodação.

Midriase direita (observada apenas no dia da entradia).

Desondens trophicas não as apresenta.

Desordens vaso-motoras ao nivel da zona anesthesica. As desordens da sudação e modifiçação da temperatura não ficaram bem esclarecidas; notava-se, porém, sensivel coloração azulada naquella zona.

A possibilidade de firmar-se um diagnostico topographico seguro reside essencialmente em dois factos, na verificação exacta das desordens da motilidade e da sensibilidade e na interpretação destes phenomenos.

As desordens da motilidade são facilmente evidenciaveis; apenas o observador terá de considerar se ellas são permanentes ou transitorias; a data do seu apparecimento e as suas modificações die intensidade com o decorrer do tempo. Schiff assentou estes principios indestructiveis :

1 - Se immediatamente ou poucos minutos ou poucas horas após a secção de uma parte da medulla verifioa-se a persistencia de uma funç̧ão, esta constitue uma prova absoluta de que esta funcçăo é independente da parte seccionada e está ligada á parte não seccionada.

2 - Se em taes condições ha deficiencia de uma funcção, não se póde ter a prova de que esta funcção pertiença á parte liesada senão quando a sua ausencia persistir ainda depois de semanas e mezes após a operação, quando finalisar-s'e a cicatrização, cessarem total- 
mente os effeitos do "shock" e o animal readquirir suas funcções normaes no gráo que the permitta a subita lesão.

Quanto aos transtornos da sensibilidade, tornam-se elles mais difficeis de localisação e apreciação exactas. Como phenomenos subjectivos que são, dependem de circumstancias individuaes e locaes. Ora é a informação do doente que tanto póde muito auxiliar o pesquizador, como desnortear-lhe a pesquiza com respostas vagas, quando se the pede a impressão causada pelos excitantes; ora são as circumstancias locaes de sensibilidade cutanea maior ou menor na zona explorada, seja pela existencia ahi dos pontos de calor, de frio, de pressão, seja pela natureza dos excitantes empregados.

Mias se de um lado a difficuldade do diagnostico é representada pelo estabelecimento preciso dos transtornos da motilidade e da sensibilidade, ha ainda a questão de serem estes devidamente interpretados.

No tocante aos phenomenos motores, afastada, á vista dos citados principios de Schiff, a hypotheıse de uma interrupção funccional, não é difficil asseverar em que altura da miedulla foram seccionadas as vias pyramidaes e as fibras radiculares; basta delimitar-se a zona paralysada. Conhecendo-se a suá innervação, póde-se concluir cum certa segurança.

Todavia a interpretação dos phenomenos sensitivos offercce maiores tropeços, «pois sendo a syndrome de Brown-Séquard o conjuncto de symptomas decorrentes de uma heminsecção medullar, segue-se naturalmente que tem grande importancia saber-se se as vias sensitivas são ou não cruzadas na medulla.

Ha profundas controversias a respeito. Entre os anatomistas, Kö1tiker affirma que as vias sensitivas são directas; Villiger e Edinger sustentam opinião justamente contraria.

Entre os experimentadores verificamos a mesma divergencia. Ao Dasso que Brown-Séquard, Schiff, Woroschilloff e Ludwig affirmam que ha o entrecruzamento, Mott, Luciani, Gotch e Horsley e outros affirmam que não.

Os estudos no homem não resolveram o caso. De um lado Dejerine. e Sottas, Pfeiffer, Schaffer, Zappert, Long e mais pesquizadores verificaram que a via sensitiva é directa. Mas vêm os factos clinicos e estabelecem o inverso; vem Van Gehuchten e affirma que as vias sensitivas percorrem o feixe de Gowers do lado opposto áquelle em que ellas se distribuem á peripheria.

Roberto Bing, no seu livro "Diagnostic topographique des lésions de l'encéphale et de la moêlle épinière assevera que, logo após seu ingresso na medulla, são separadas as fibras de conducção das quatro qualidade primordiaes da sensibilidade. A sensibilidade tactil segue tanto pelas fibras curtas como pelas fibras longas do systema 
radicular posterior, isto é; tanto pelas fibras homologes. (directas ou tambem chamadas tautomeras) como pelas fibras do lado opposto ou cruzadas (heteromeras); e, finalmente, pelo feixe espinho-thalamico. A sensibilidade thermica e a sensibilidade dolorosa seguem pelias fibras radiculares posteriores curtas (que terminam nas cellulas dos córnos posteriores) e depois, pelos seus neuronios de segunda ordem, passam para o cordão lateral do la do opposto para aloançar o thaiamo. A sensibilidade profunda segue uma dupla via, ambas directas: a) chega ao thalamo e ao cerebro pelas fibras radiculares posteriores longas e cordões posteriores; b) pelas fibras ra lieulares posteriores rnedianas, columnas de Clarke e feixe cerebelloso directo, chega ao cerebello.

Para Bing, portanto, as sensibilidades thermica e dolorosa têm vias cruzadas; a sensibilidade tactil, parte directa e parte cruzadia; a sensibiiidade profunda segue por vias directas.

Petrén, baseado em quasi uma centena de casos de hemi-secção medullar produzica por instrumentos cortantes, enuncia que as sensibilidades thermica e dolorosa seguem um trajecto cruzado; a tactil, directo; a profunda, tanto, um trajecto cruzado (feixe de Flechsig do lado opposto) como outro directo (cordão posterior).

Em a nossa observação o iogar de entrada do instrumento lesante encontra-se á esquerda; quer dizer: do lado contrario aos phenomenos motores.

$\mathrm{Ha}$ em clinica observações de ferimentos produzidos do lado opposto áquelle em que surgiram as perturbações da motilidade. Foram estes cas,os interpretados, seja como resultantes de lesão das vias pyramidaes antes do seu entrecruzamento bulbar, scja como casos em que os phenomenos motores surgiram não por uma secção pyramidal mas apenas por uma causa de compressão - uma hemorrhagia, por exemplo.

No nosso observado a explicação deve ser outra. A marca da entrada encontra-se á esquerda; mas, segundo conta o proprio doente, o aggressor produziu o ferimento-collocando-se a sua direita. Quer dizer que, estando o aggredido em posição curvada para o sólo, ha toda a probabiiidade de que a 'direcção do ferimento seja da esquerda para a direita (com o movimento do braço do aggressor) e embora o logar de penetração da faca na pelle fosise á esquerda, a parte attingidia da medulla foi a direita. 


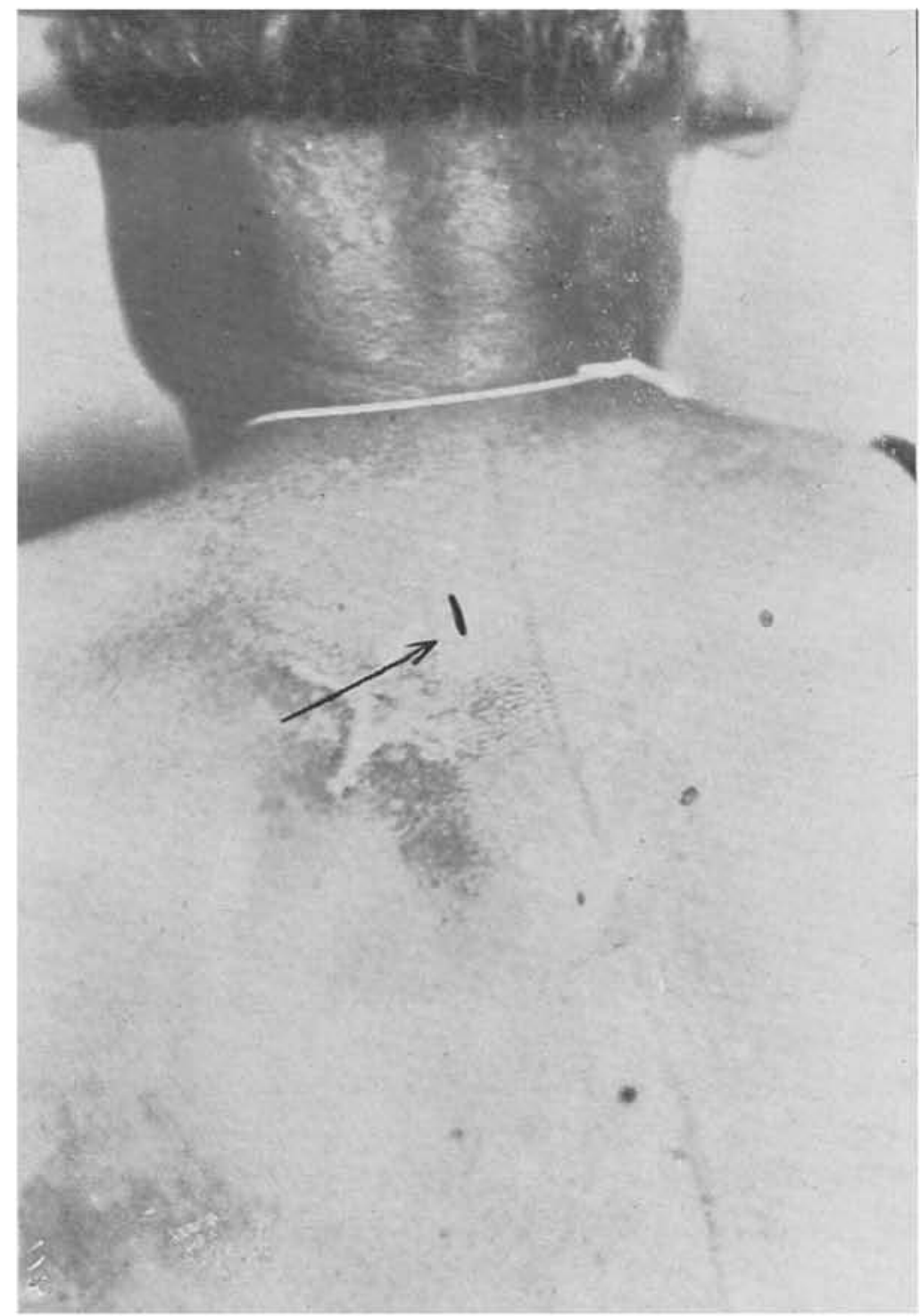

FIG. I

A seta indica o ponto de penetração da faca 



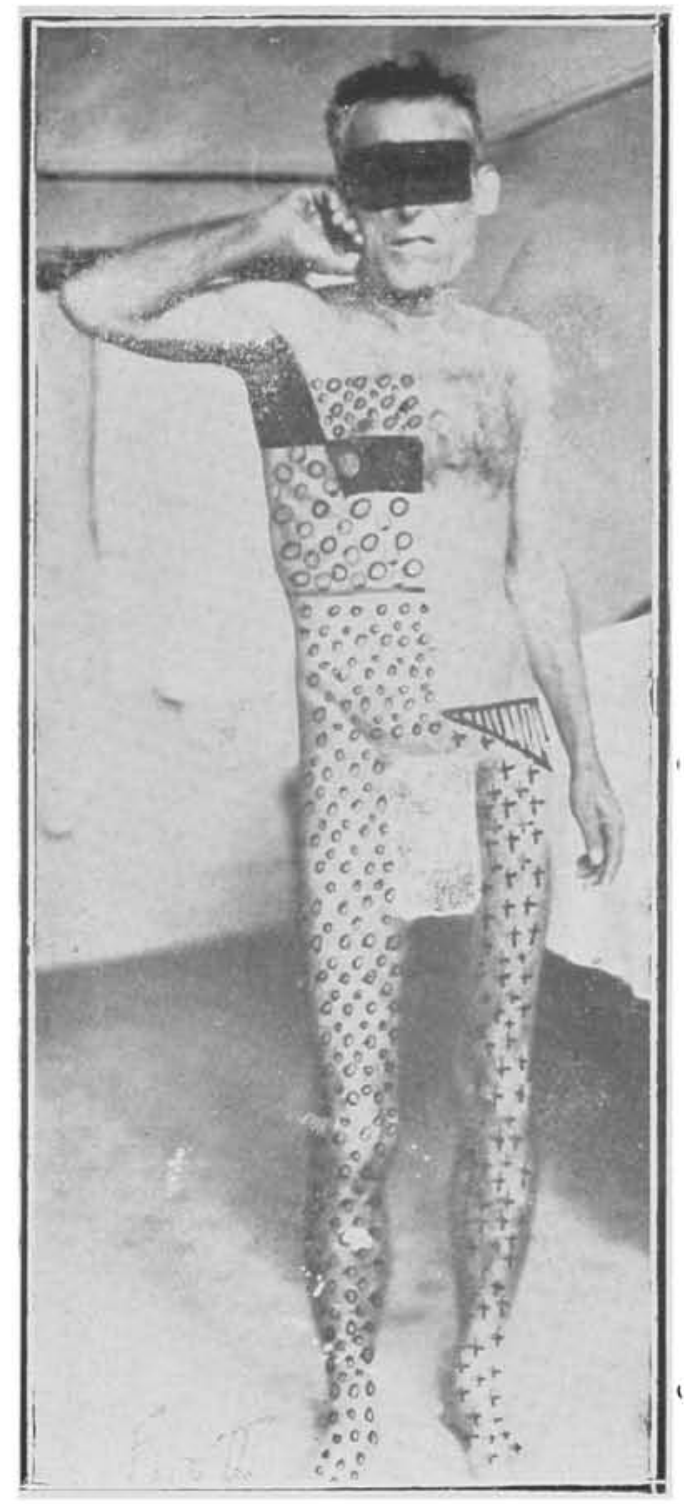

FIG. II

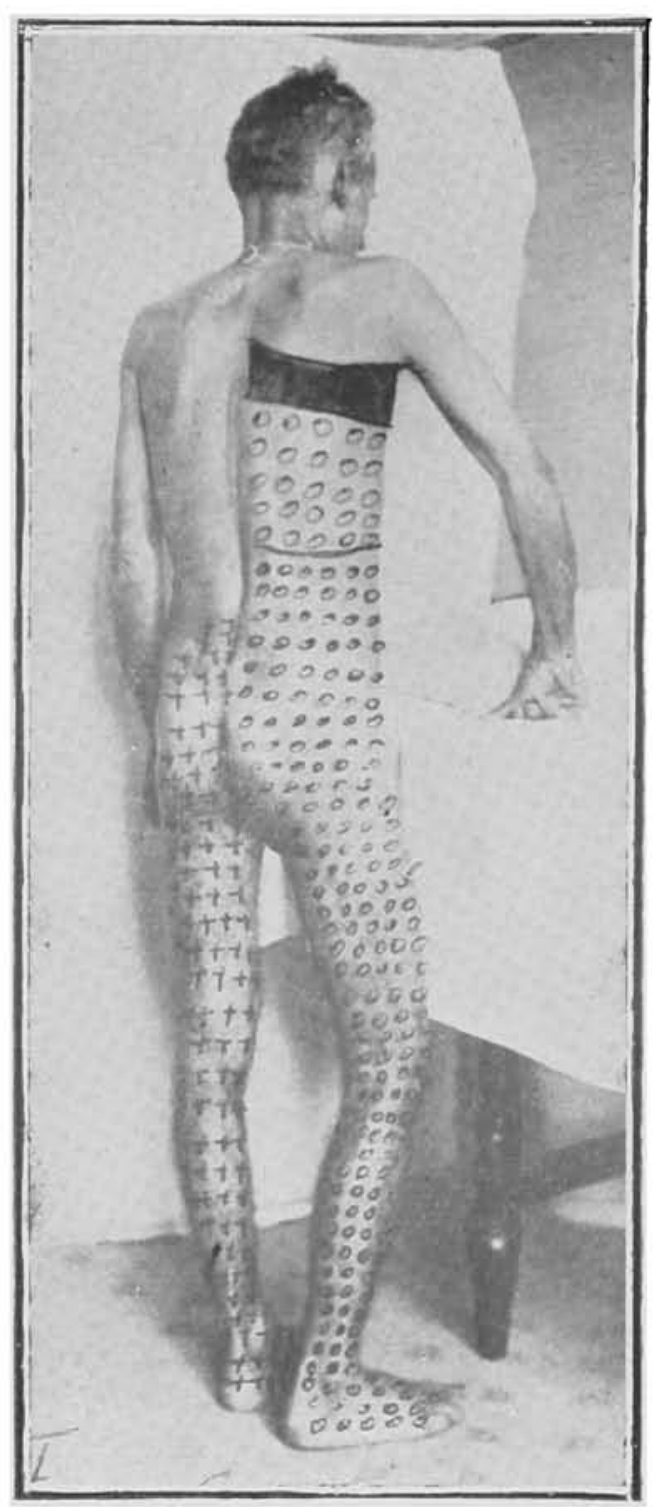

FIG. III

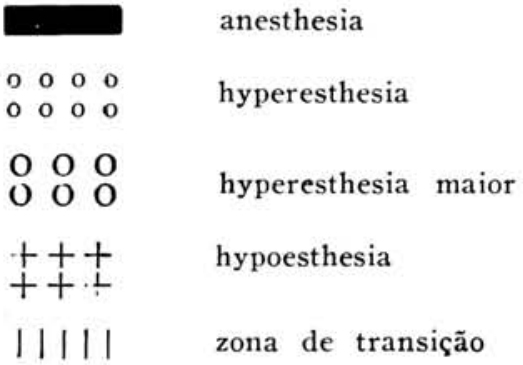



Nao ha, pois, anomalia da syndrome; os phenomenos motores, como de regra, são directos, ao passo que os sensitivos são cruzados.

De accôrdo com o enunciádo a respeito das vias motoras e sensitivas, podemos concluir no nosso caso que houve uma hemilesão medullar do lado direito, entre a 3.' e 4." vertebras dorsaes, tendo attingido o $4 .^{\circ}$ nervo dorsal e os feixes lateraes da medulla. Dahi a ancsthesia no dominio da innervação do $4 .^{\circ}$ dorsal, a hemiparaplegia direita e as perturbações sensitivas.

E' innegavel que o $4 .^{\circ}$ dorsal direito foi attingido, porque na zona por elle innervada ha anesthesia cutanea, persistintlo a sensibilidade profunda - o que é proprio da anesthesia não de origem medullar, mas peripherica. Evidentemente o nervo foi lesado na visinhança de sua emergencia da medulla.

Quanto ás perturbações sensitivas, vemos que effectivamente o nosso caso mostra serem cruzadas as vias das sensibilidides thermica e dolorosa.

No que se refere ás sensibilidades tactil e profunda, presentes no nosso doente, nàa podemos dizer de seguro, pois o nosso exame foi feito muitos dias após o ferimento e-não nos é licito affirmar se ellas foram desde o inicio poupadas ou si já se haviam restabelecido na êpoca da nossa pesquiza. Todavia, a admittir que ellas fossem sempre presentes após o ferimento, é claro que a conclusão legitima no caso é de que a sensibilidade tactil e a sensibilidade profựda têm simultaneamente vias directas e cruzada na medulla.

$O$ interesse da observação que apresentamos tambem está na zona anesthesica, de limites um tanto caprichosos no thorax, e no facto de os phenomenos sensitivos cruzados se apresentarem em limite muito inferior áquelle em que encontramos a anesthesia e a hyperesthesia homologas.

A circumstancia da data do nosso exăme (cerca de um mez após o ferimento) impede-nos de bordar uma interpretação bem fundada, assim como de alongar-nos em outras considerações que um caso destes comporta.

Năo podiemos entretanto deixar de chamar a attenção para um ponto: o nosso caso não constitue excepção ás conclusões dos estudos de Petrén. Enquadra-se perfeitamente em um dos tres grupos que compôem os 94 casos observados por este autor (v. Revue Neurologique, 1911):

Grupo I: Casos em que existe, no principio, perturbação da motilidade só de um lađo, e em que a anesthesia cruzada não affecta senão a sensibilidade thermica e dolorosa, deixando intacta a sensibilidade ractil (39. casos).

Grupo II: Casos em que existem no principio perturbações da motilidade só de um lado, mas em que a anesthesia affecta todas as formas da sensibilidade cutanea, isto é, a sensibilidade tactil (24 casos). 
Grupo III: Casos em que existem no principio perturbações dos dois membros inferiores e em que a anesthesia cutanea se comporta como no grupo II (31 casos). Não existe nenhùm caso de paralysia dos dois membros inferiores com anesthesia dissociada do typo syringomyelico (como no grupo I).

No mesmo trabalho de Petrén vamos encontrar a respeito do sentido muscular (impressão das attitudes segmentarias) que:

a) No grupo I ha casos sem perturbações do sentido muscular e ha outros com estas.perturbações, do lado da lesão, - mas não existe um só caso com taes perturbações bilateraes.

b) No grupo II, perturbações desse sentido do lado da lesão constituem a regra; a bilateralidade das perturbações não foi nem uma só vez demonstradia.

c) No grupo III encontram-se muitos casos com perturbações do sentido muscular sómente do lado da lesão principal da medulla, e muitos casos com tares perturbações de ambos os lados. Pelo contrario, não ha um só caso nos grupas II e III com sentido muscular normal.

Dahi conclue Petrén que a sensibilidade profunda segue tanto um tnajecto directo (cordão posterior) cơmo um outro, cruzado (feixe de Flechsig do lado opposto).

O quadro das syndromes de Brow-Séqnard é inteiramente dominado pelos phenomenos motores e sensitivos. Entre aquelles é a paralysia espasmodica com contractura mais ou menos accentuada: nestes, a hypo e as hyperesthesias. Para melhor exposição passaremos $\mathrm{cm}$ exame succinto os phenomenos encontrados:

1. $\left.{ }^{\circ}\right)$ Phenomenos motores: A extensão e a sédie das desordens da motricidade dependem, antes de mais nadia, da altura da lesão. Queremos nos referir aos ferimentos mais altos, logo abaixo do occipital, que acarretam quasi sempre paralysia cruzada, em virtude de ser attingido o feixe pyramidal antes da decussão. São ainda os traumatismos altos que trazem modificações pupillares e mesmo da rima palpebral. No nosso caso devemos interpretar a midriase direita como phenomeno funccional decornente de transitoria cómpressão á distancia do centro cilio-espinhal.

A paralysia francamente espasmodica algum tempo após a lesão póde comtudo ser flacida nos primeiros momentos, podendo por isso dar origem a um diagnostico de secção total, pela ausencia completa de reflexos. Para logo, porém, o membro correspondente é tomado de 
parezia ou paralysia, e a percussão dos tendões dá logar a grandes movimentos. Outra eventwalidade que merece ser destacada e lembrada todas as vezes que examinamos doentes dessa natureza, são as suppurações das feridas racheanas, que haviam dado origem a syndrome de hemi-secção. Nesisa emergencia, os reflexos desapparecem de um dia para outro, podendo ou não coincidir com o augmento de temperatura etc

Esses factos devem estar bem presentes, para que evitemos o erro de diagnostico a principio, ou vacillaçõ.es depois que o houvermos estabelecido.

$2 .^{\circ}$ ) Phenomenos sensitivos: As perturbações da sensibilidade são as mais importantes e serão divididas em perturbações sensitivas do mesmo lado da lesão e do lado opposto.

Do mesmo lado - Sensibilidade profunda: A sensibilidade baresthesica geralmente desapparece, se bem que não pequeno é o numero dos casos em que ella se manteve integra. Como já vimos posteriormente, em o nosso caso ella estava presente quando praticámos os nossos exames. O mmesmo se diga da sensibilidade ossea, articular e da noção segmentaria, todas presentes no nosso doente, mas que podem estar compromettidas mais ou menos profundamente. Póde tambem ser observado separadamente o desapparecimento de uma ou outra dessas senisibilida des.

Sensibilidade superficial: Encontram-se - como no nosso caso faixas de hyperesthesia e anesthesia. Estra abarca o hemithorax correspodente, em forma de uma faixa de alguns centimetros cujo limite superior geralmente é marcado pela lesão. A anesthesia ahi é absoluta : o arrancamento de um pello, a picada profunda de um alfinete, um tudo contendo agua muito quẹnte, não despertam a menor reacção.

Fraz-se mistér insistir sobre os contornos verdadeiramente bizarros da faixa anesthesica por nós verificada. Démo-la de principio como resultado de informes errados, mas as dezenas de pesquizas feitas depois confirmaram categoricamente o primitivo traçado. Trata-se positivamente de trajecto ou anastomose anormal dos nervos corespondentes.

Continuaremos, como vimos fazendo, o cotejo dos dados de um caso ideal - se pudessemos assim dizer, com o concreto, que são o nosso e os mais encontradiços, se bem que a verdade no-la dá Miguel Couto, quando, referindo synopse em pathologia, diz: "pequeno para conter as infinitas particularidades realisaveis em certos casos, grande para um só caso preenchêl-o"

Assignalamos nas photographias e na observação a intensidade vária da hyperesthesia. 
A fáixa anesthesica fica geralmente comprehendida entre uma faixa hyper-: esthesica superior (que já deu-motivo a muita controversia) e outra inferior. que vae até a extremidade do membro. Esta, em nosso caso, é divisivel em duas partes distinctas consoante registamos na observação.

Fazem ainda parte dos symptomas directos os phenomenos vaso-motores. acarretando ora a cyanose, ora a hyperthermia acompanhada de sudação.

Oeccorrem posteriormente as perturbações trophicas, que um de nós teve opportunidade de verificar na clinica do Prof. Rocha Vaz: foi um caso no qual a atrophia dos musculos da coxa, e principalmente da perna, estabelecia enorme contraste com a compleição athletica do tronco e dos membros superiores. Esise mesmo doente apresentava uma descamaçẫo pronunciada dos membros inferiores e da face plantar dos pés.

No lado opposto á lesão dominam os phenomenos sensitivos, que consistem em anesthesia superficial.

No caso que deu origem a esta publicação existia uma hypoesthesia ligeira occupando uma área correspondente a todo o membro inferior esquerdo e parte inferior do abdomem, consoante se vê em gravura.

Desordens das visceras thoraxicas existem nos processos altos da medulla; mais frequentes são as perturbações de esphincteres, como succedeu no nosso caso.

Muito mais raras são certas modalidades especiaes em que ha modificações profundas do quadro symptomatologico. Entre essas merecem ser lembradas a dissociação syringomyelica e a dupla syndrome de BrozenSéquard.

Aquella, unicamẹte pelos symptomas em si, não poderá ser differenciada da syringomyelia; faz-se mistér comparar os campos anesthesicos, que têm disposição radicular na syringomyelia.

Raymond affirma, porém, serem esses factos muito mais frequentes do que geralmente se acredita, o que está em desaccôrdo com a observação de outros autores.

Pàra Van Gehuchten a dissociação syringomyelica é produzida pela lesão do feixe de Gowers, ao passo que Brissaud acreditava na hemi-secção incompleta que não houvesse attingido os fieixes posteriores.

A dupla syndrome de Brown-Séquard é produzida pela associação de duas lesões em alturas differentes. Esta eventualidade nunca offërece, porém, como é bem de ver, uma symptomatologia nitida. Ha superposição das desordens, dando assim paraplegia espamosdica mais ou menos equivalente, emquanto que as desordens sensitivas são confusas, principalmente nos membros. 
Quaes os processos capazes de provocar o apparecimento da syndrome de Brown-Séquard? Poderão ser descriptos em dois grandes grupos:

a) Causas traumaticas: ferimentos por instrumento cortante, ferimento por arma de fogo, fracturas, luxações, commoções, etc.

b) Causas inflammatorias, neoplasicas etc.: syringomyelia, meningites racheanas, myelites difusas, arthrites, blastomas, myelites syphiliticas, gommas meningeanas, mal de Pott etc.

Os processos syphiliticos, em geral, são excluidos pela feição muito propria que apresentam: exacerbações nocturnas, outros indicios clinicos ou signaes de maior oerteza mercê de reacções serologicas. Ademais, o estacionamento e mesmo as melhoras mais ou menos accentuadas, com o tratamento especifico, constituem a contra-prova.

Para estabelecer a differenciação da syringomyelia, já o dissemos, basta attentar para a distribuição das zonas thermoanesthesicas e phenomenos motores. Quanto ás meningites, tumores. etc., os exames cytoscopico, chimico e biologico do liquido cephalo-racheano, firmam definitivamente o diagnostico.

Pelo exame superficial que acabamos de fazer, aquilata-se bem das duvidas que podem surgir algumas vezes para chegarmos scientificamente e sem nos valermos da casuistica, a um diagnostico etiologico certo, em contraposição ao diagnostico da syndrome em si, que não offerece difficuldades maiores.

O prognostico das syndromes de Brown-Séquard (por traumatismos) é bom. A funcção tem quasi uma restitutio ad integram, excepção feita para os casos de ferimentos contaminados, evoluindo para a suppuração, em que é sombrio, maximé quando, após um periodo de completo silencio, se exteriorisa violentamente, como num caso que nos conta Miguel Couto.

$\mathrm{O}$ caso de Rocha Vaz, de que já fallámos, só conseguia andar decorridos quasi dois annos de sua entrada para a enfermaria. E só o fazia arrimando-se nas paredes ou na bengala.

A benignidade do prognostico da syndrome de Brown-Séquard depende exclusivamente da causa que the deu origem.

Muito discutida é a questão do mecanismo da reintegração das funcções sensitivas e motoras. Não entraremos na questão da regeneração das fibras. Lembraremos apenas que a restitutio da funcção não é effeito della. A proliferação observada não é das fibras, segundo uns - que essa, por mais abundante, não póde flanquiear a barreira de tecido fibroso interposta nos labios da seç̧ão. 
Os outros mecanismos descriptos com o fim de explicar o prognostico benigno tiveram vida ephemera.

Em conclusão : o "doente deve escapar da morte - diz Miguel Couto póde escapar perfeito, mas a regra é ficar com uma paralysia incompleta, acompanhada de espasmos e atrophia..."

Maio de 1923.

DR. FELIPE FIGLIOLINI.

DR. FELICIO CINTRA DO PRADO.

\section{ANNUNCIEM NA "REVISTA DE MEDICINA"}

Mediante pedido enviamos tabellas de preços e prestamos promptamente quaesquer outras informações.

Certifico que tenho empregado com bons resultados, em minha clinica o reconstituinte VIDAN em cuja formula se associam o hypophosphito de calcio, glycerophosphato de magnesio, glycerina, kola e arsenico.

a) DR. CELESTINO BOURROUL 